\title{
Longitudinal Analysis of Severe Anxiety Symptoms in the Last Year of Life Among Patients With Advanced Cancer: Relationships With Proximity to Death, Burden, and Social Support
}

\author{
Siew Tzuh Tang, DNS ${ }^{a, b, c}$; Jen-Shi Chen, MDc; Wen-Chi Chou, MD; ; Wen-Cheng Chang, MD; \\ Chiao-En Wu, MDc; Chia-Hsun Hsieh, MDc; Ming-Chu Chiang, MS ${ }^{\text {b }}$; and Mei-Ling Kuo, MS ${ }^{\text {b }}$
}

\begin{abstract}
Background: Temporal changes in the prevalence of anxiety disorders/symptoms for patients with cancer at the end of life (EOL) remain unclear. This study was undertaken to describe changes in the prevalence of severe anxiety symptoms and to identify its correlates in the last year of life for patients with cancer. Methods: A convenience sample of 325 patients with cancer was followed until death. Severe anxiety symptoms were identified as anxiety subscale scores of 11 or greater on the Hospital Anxiety and Depression Scale. Longitudinal changes in and correlates of severe anxiety symptoms were examined from demographics, disease-related characteristics, disease burden, perceived burden to others, and social support using multivariate logistic regression modeling with generalized estimating equations. Results: The prevalence of severe anxiety symptoms increased as death approached (18.6\%, 21.9\%, 26.7\%, and 33.4\% at 181-365, 91-180, 31-90, and 1-30 days before death, respectively). However, after controlling for covariates, this temporal increase was not significant. The prevalence of severe anxiety symptoms was not associated with fixed demographics and disease-related characteristics, except for diagnosis and metastatic status, but was significantly higher in patients with cancer with high physical symptom distress, severe depressive symptoms, high perceived burden to others, and strong perceived social support. Conclusions: Severe anxiety symptoms were not associated with time proximity to death per se but were related to factors modifiable by high-quality EOL care. Clinicians may decrease the likelihood of severe anxiety symptoms at EOL by adequately managing physical and depressive symptoms and lightening perceived burden to others for patients strongly connected with their social network to improve their psychological well-being.
\end{abstract}

J Natl Compr Canc Netw 2016:14(6):727-734

\section{Background}

Anxiety in patients with advanced/terminal cancer is a dynamic response to uncertainty about their impending mortality, perceived threats of physicalpsychosocial-spiritual suffering, and concerns about

From a Chang Gung University, School of Nursing, Tao-Yuan City, bepartment of Nursing, Kaohsiung Chang Gung Memorial Hospital, Kaohsiung City, and 'Division of Hematology-Oncology, Chang Gung Memorial Hospital, Linkou, Tao-Yuan City, Taiwan.

Submitted October 25, 2015; accepted for publication March 21, 2016.

This study was funded by the National Science Council (NSC99-2628-B182-031-MY2), National Health Research Institute (NHRI-EX105-10208PI), and Chang Gung Memorial Hospital (BMRP888). None of the funding sources had any role in designing and conducting the study: collecting, managing, analyzing, and interpreting the data; or preparing, reviewing, or approving the manuscript. The authors have disclosed that they have how their death will affect loved ones. ${ }^{1,2}$ These patients' prevalence of anxiety disorders $(7.6 \%-18.0 \%)^{3-5}$ and anxiety symptoms $(12.4 \%-48 \%)^{1,6-15}$ is substantial. Nonetheless, anxiety has not been as extensively studied in psycho-oncology research as depression,,$^{1,3}$ but

no financial interests, arrangements, affiliations, or commercial interests with the manufacturers of any products discussed in this article or their competitors.

Author Contributions: Study concept and design: Tang, Chen, Chou, Chang, Wu, Hsieh, Chiang, and Kuo. Acquisition of data: Tang, Chen, Chou, Chang, Wu, Hsieh, Chiang, and Kuo. Analysis and interpretation of data: Tang. Drafting of manuscript: Tang. Final approval of manuscript: Tang, Chen, Chou, Chang, Wu, Hsieh, Chiang, and Kuo.

Correspondence: Siew Tzuh Tang, DNS, Chang Gung University, School of Nursing, 259 Wen-Hwa 1st Road, Kwei-Shan, Tao-Yuan, Taiwan, 333.

E-mail: sttang@mail.cgu.edu.tw 
Tang et al

represents a unique problem in its own right. Anxiety disturbances are associated with depression, ${ }^{5}$ spiritual suffering ${ }^{16,17}$ (eg, perceived burden to others), ${ }^{5}$ impaired quality of life (QOL), ${ }^{12}$ and suicidal ideation/desire in patients with advanced cancer. ${ }^{8,18}$

However, studies exploring anxiety-symptom/disorder prevalence in patients with advanced cancer are predominantly cross-sectional., ${ }^{1,-10,12-15}$ Furthermore, results are conflicting on longitudinal changes in anxiety symptoms at end of life (EOL). Anxiety symptoms/ disorders are generally suggested to be exacerbated at EOL. ${ }^{2,11}$ However, anxiety-disorder prevalence in a 94-study meta-analysis did not differ between palliative and non-palliative care settings, where palliative care setting was a proxy for EOL. ${ }^{3}$ Similarly, cancer patients' rates of mental disorders (including anxiety disorders) have not increased significantly as death approached. ${ }^{19}$ Such conflicting results may stem from how anxiety symptoms were measured. Among the few studies on changes in anxiety-symptom/disorder prevalence in patients with terminal cancer, only one study directly assessed anxiety using a single item, ${ }^{11}$ and others estimated time proximity to death by treatment setting ${ }^{1,3}$ or divided time proximity to death by cross-sectional data. ${ }^{19}$ Therefore, temporal changes in anxiety-symptom/disorder prevalence during cancer patients' EOL remain unclear. Furthermore, knowledge about anxiety symptoms in terminally ill patients with cancer is from Western countries. ${ }^{1,4-15}$ The importance of understanding severe anxiety symptoms in terminal Asian patients is underscored by different factor structures across diverse cultures for the Hospital Anxiety and Depression Scale (HADS; the most frequently used instrument for measuring anxiety symptoms). ${ }^{20}$ Understanding the factors associated with severe anxiety symptoms in terminally ill patients with cancer may help clinicians identify at-risk patients, enabling early referral and interventions for this profound psychological morbidity. ${ }^{21}$ Therefore, we explored changes in severe anxiety-symptom prevalence and identified its determinants in Taiwanese patients with cancer in their last year of life.

\section{Methods}

\section{Design and Sample}

This longitudinal analysis extends a study of factors influencing QOL in terminally ill Taiwanese patients with cancer during their dying process ${ }^{22}$ by increas- ing the follow-up time through December 2013. Detailed methods were published.22 Adult patients with cancer were referred to research assistants by their oncologist who declared them as terminally ill when their disease was progressive and unresponsive to curative treatments. The Chang Gung Memorial Hospital ethics committee approved the research protocol. All participants signed informed consents.

\section{Measures}

Data were collected on anxiety symptoms and 5 groups of variables (demographics, disease-related characteristics, disease burden, perceived burden to others, and social support) potentially predicting participants' severe anxiety symptoms..$^{21}$ Except for demographics and disease-related characteristics, all variables were assessed approximately every 2 weeks until participants declined to continue or died.

Anxiety symptoms were measured by the 7 -item HADS-anxiety subscale (HADS-A).23 Although HADS is the most widely used tool for assessing psychological morbidity in patients with cancer undergoing palliative care ${ }^{24}$ it is appropriate for screening, not for diagnosis. ${ }^{25}$ The total HADS-A score range is 0 to 21 ; higher scores indicate greater anxiety. We identified severe anxiety symptoms by HADS-A scores 11 or greater, ${ }^{23}$ despite debate about the optimal cut point. ${ }^{26}$

Demographics included sex, age, marital status, educational level, and religious affiliation. Diseaserelated characteristics included diagnosis, metastatic and comorbidity status, and postdiagnostic survival at enrollment. Postdiagnostic survival, the time between initial cancer diagnosis and enrollment, was categorized into 6 or less, 7 to 12,13 to 24 , and 25 or more months.

Disease burden was represented by physical and psychological symptom distress and functional dependence. Physical symptom distress was measured by the 13-item Symptom Distress Scale (SDS), ${ }^{27}$ which assesses common symptoms in patients with cancer. The score range is 13 to 65 ; higher scores indicate greater distress. Functional dependence was measured by the 10-item Enforced Social Dependency Scale (ESDS). ${ }^{28}$ The total ESDS score range is 10 to 51 ; higher scores reflect greater dependence on help for personal and social functioning. Total mean (SD) SDS and ESDS scores for patients with advanced cancer were $17.6(5.9)$ to $32.74(10.75)^{29}$ and 20.0 to $43.3,3^{30}$ respectively. Chinese-version 
SDS and ESDS internal consistencies (Cronbach's alphas) in this study were 0.86 and 0.92 , respectively. Chinese-version SDS and ESDS construct validities were established by their scores' positive associations with depressive symptoms ${ }^{31}$ and negative associations with $\mathrm{QOL}^{22}$ in Taiwanese patients with cancer during their dying process. Psychological symptom distress (depressive symptoms) was measured by the HADS depression subscale (HADS-D). ${ }^{23}$ The total HADS-D score range was 0 to 21 , with scores of 11 or greater considered severe depressive symptoms. ${ }^{23}$

Perceived burden to others, deriving from patients' dependence on others for assistance, ${ }^{32}$ was measured by the 10-item Self-Perceived Burden Scale (SPBS). ${ }^{32}$ Receiving EOL care can lead to feeling that one is creating physical, emotional, social, and economic hardships for one's family without opportunities to restore the balance between receiving and giving help. ${ }^{33}$ The SPBS assesses guilt, dependence, and resentment, as well as worries that caregiving may interfere with the caregiver's life, affecting his/her health. SPBS items are rated on a 5-point scale from 1 (none of the time) to 5 (all of the time); higher scores indicate greater perceived burden to others. SPBS scores of 20 or greater distinguished patients with advanced cancer with high or low perceived burden to others. ${ }^{34}$ Mean (SD) SPBS scores for patients with advanced cancer were 18.91 $(6.63)^{34}$ to 19.28 (7.12). ${ }^{35}$ SPBS psychometric properties have not been formally tested in Taiwan, but SPBS scores were negatively associated with QOL levels ${ }^{22}$ and positively associated with severe depressive-symptom likelihood ${ }^{31}$ in terminal Taiwanese patients with cancer. Cronbach's alpha for the SPBS in this study was 0.91 .

Social support was measured using the 19-item Medical Outcomes Study Social Support Scale (MOS-SSS).$^{36}$ MOS-SSS items measure emotional, informational, tangible, and affectionate support, as well as positive social interaction. A total raw score is computed, then transformed into a $\mathrm{O}$ - to 100 -point scale. Higher scores indicate better perceived social support. The normative mean (SD) MOS-SSS score from 2,987 chronically ill adult patients was 70.1 (24.2).$^{36}$ Cronbach's alphas in this study were 0.77 to 0.95 . The Taiwanese-version MOS-SSS had a 2-factor structure; its construct validity was supported by MOS-SSS scores' positive association with spirituality. ${ }^{37}$

\section{Analysis}

Participants' characteristics were analyzed by descriptive statistics. Chi-square tests and multivariate ANOVA were used to compare baseline characteristics and anxiety symptoms among participants who died, withdrew, and were still alive at the end-ofstudy follow-ups. Group differences were determined using Tukey's honest significant different (HSD) post hoc test for studentized range statistic, thus controlling for type I errors. To explore longitudinal changes in severe anxiety-symptom prevalence in participants' last year, temporal proximity to patient death - the period between death and assessmentwas categorized as periods of 1 to 30,31 to 90,91 to 180 , and 181 to 365 days, which are conventional periods for estimating survival of patients with cancer. ${ }^{38}$ Because participants could contribute multiple data to study variables (anxiety symptoms, disease burden, perceived burden to others, and social support) during a specific period (eg, 181-365 days), all data for each variable were averaged across that period in final analyses.

The association of independent variables with severe anxiety symptoms was assessed using multivariate logistic regression modelling with the generalized estimating equation (GEE). ${ }^{39}$ The GEE uses robust standard error estimates to account for within-subject correlations ${ }^{39}$ of HADS-A scores during follow-up and to accommodate variable numbers of follow-ups, inconsistent intervals for subsequent data collections, and missing data. ${ }^{39}$ The regression estimate for each independent variable was exponentiated to transform into an adjusted odds ratio (AOR) with a $95 \% \mathrm{CI}$.

\section{Results}

Of 433 eligible patients, 380 were enrolled $(87.8 \%$ participation) (Figure 1). Characteristics of patients who did and did not participate cannot be compared because of restricted access to information about those who refused to participate. Patients who died during the study $(N=325)$ comprised the final sample (Figure 1). These patients and those who withdrew or were still alive generally did not differ significantly at baseline. However, fewer patients who withdrew from the study experienced severe depressive symptoms (HADSD scores $\geq 11$ ) than those who died or were still alive (23.8\%, 54.1\%, and 50.0\%, respectively; $P=.03$ ). 
Tang et al

Participants were primarily male, older than 56 years, married, and Buddhists/Taoists (Table 1). Half the patients $(50.1 \%)$ had stomach, liver, and pancreatic cancer. At baseline, participants' physical symptom distress, functional dependence, and perceived social support were within ranges reported for patients with advanced cancer ${ }^{29,30,36}$ (Table 1), but self-perceived burden to others was greater. ${ }^{34,35}$ After enrollment, participants survived 153.12 days (SD, 162.40; median, 94.0; range, 5-667) and completed on average 7.65 follow-up assessments (SD, 7.95; median, 5; range, 1-34).

Severe anxiety-symptom prevalence (HADS-A score $\geq 11$ ) at 181 to 365,91 to 180,31 to 90 , and 1 to 30 days before death was $18.6 \%, 21.9 \%, 26.7 \%$, and $33.4 \%$, respectively. Unadjusted GEE analysis showed that the closer the time to patient death, the higher the likelihood of severe anxiety symptoms (odds ratio, 1.34 [95\% CI, 0.93, 1.92], $P=.11$; 1.84 [95\% CI, 1.26, 2.69], $P=.002$; and 2.70 [95\% CI, $1.79,4.08], P<.001$ at $91-180,31-90$, and $1-30$ days, respectively, relative to 181-365 days before death). After controlling for covariates in multivariate GEE analysis, severe anxiety-symptom prevalence did not change significantly as death approached (Table 2).

Participants' likelihood of severe anxiety symptoms was not significantly associated with demographics and disease-related characteristics, except for diagnosis and metastatic status (Table 2). $\mathrm{Pa}$ tients with lung cancer and metastatic disease were
0.43 (95\% CI, 0.21, 0.89) and 0.56 times (95\% CI, $0.36,0.87)$ less likely to experience severe anxiety symptoms than those with other diagnoses and without metastasis, respectively.

In contrast, the likelihood of severe anxiety symptoms was significantly associated with disease burden, perceived burden to others, and social support (Table 2). Participants were significantly more likely to have severe anxiety symptoms at EOL if they had severe physical symptom distress (AOR, 1.12 [95\% CI, 1.09, 1.14] with each unit increase in SDS score) and severe depressive symptoms (AOR, 4.55; 95\% CI, 2.94, 7.14). Similarly, the likelihood of severe anxiety symptoms was 1.75 times (95\% CI, $1.321,2.31)$ greater for patients with high perceived burden to others than for those with little/no corresponding perceived burden. The likelihood of severe anxiety symptoms was higher for patients with better social support (AOR, 1.02 [95\% CI, 1.01, 1.03] with each unit increase in MOS-SSS score).

\section{Discussion}

Prevalence of severe anxiety symptoms (HADS-A scores 211 ) increased as death approached. However, after controlling for covariates, severe anxietysymptom prevalence did not change significantly in patients' last year, but was associated with disease burden, perceived burden to others, and social support.

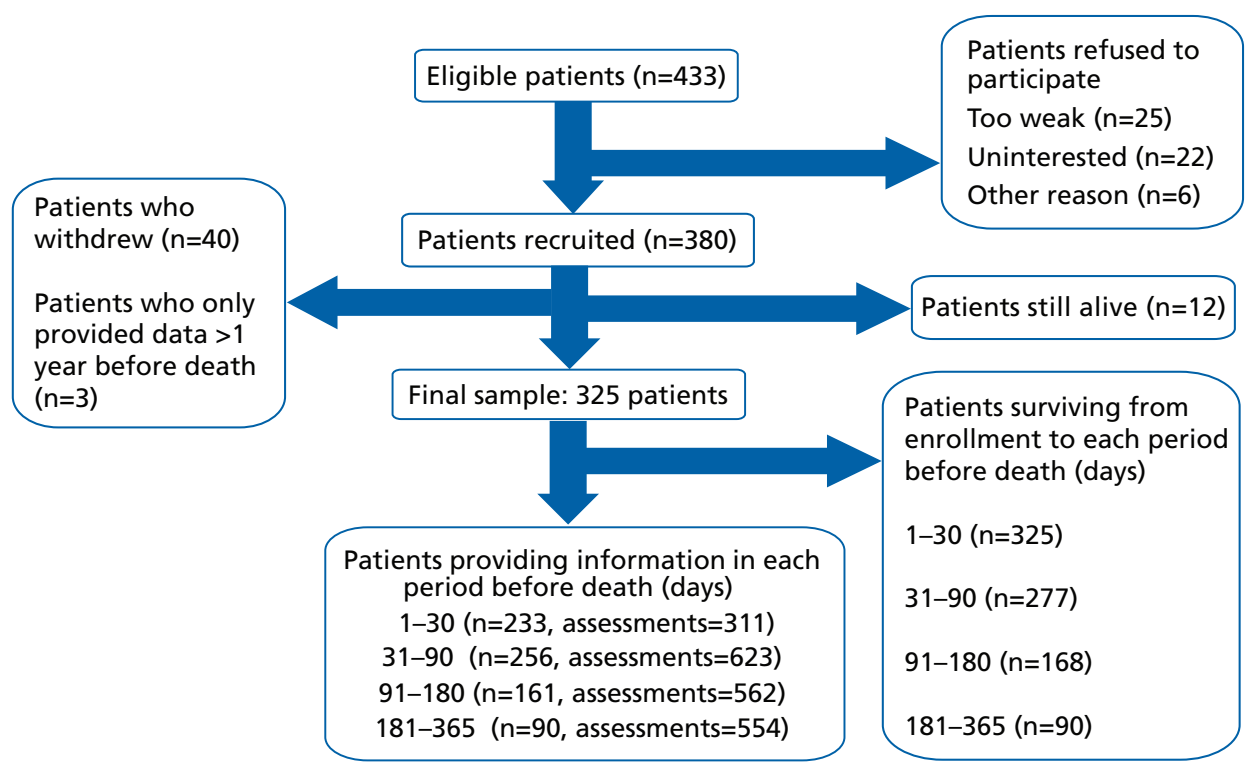

Figure 1. Participant flow chart. 
Anxiety Symptoms at End of Life

\begin{tabular}{|c|c|c|c|c|c|c|}
\hline \multicolumn{2}{|c|}{ Parameter } & $\mathbf{n}$ & $\%$ & Parameter & $\mathbf{n}$ & $\%$ \\
\hline \multirow[t]{3}{*}{ Sex } & & & & \multicolumn{3}{|l|}{ Cancer site } \\
\hline & Male & 187 & 57.5 & Stomach & 61 & 18.8 \\
\hline & Female & 138 & 42.5 & Liver & 54 & 16.6 \\
\hline \multicolumn{4}{|c|}{ Age $(y)$} & Pancreas & 49 & 15.1 \\
\hline & $\leq 45$ & 50 & 15.4 & Head and neck & 33 & 10.2 \\
\hline & $46-55$ & 85 & 26.2 & Lung & 32 & 9.9 \\
\hline & $56-65$ & 90 & 27.7 & Esophagus & 21 & 6.5 \\
\hline & $>65$ & 100 & 30.8 & Colon/Rectum & 20 & 6.2 \\
\hline \multicolumn{4}{|c|}{ Marital status } & Other & 55 & 16.9 \\
\hline & Married & 250 & 76.9 & \multicolumn{3}{|l|}{ Metastasis } \\
\hline & Unmarried & 57 & 17.5 & Yes & 244 & 75.1 \\
\hline & Missing & 18 & 5.5 & No & 80 & 24.64 \\
\hline \multicolumn{4}{|c|}{ Religious affiliation } & Missing & 1 & 0.3 \\
\hline & None & 72 & 22.2 & \multicolumn{3}{|l|}{ With chronic disease } \\
\hline & $\begin{array}{l}\text { Christianity/ } \\
\text { Catholicism }\end{array}$ & 29 & 8.9 & Yes & 196 & 60.31 \\
\hline & $\begin{array}{l}\text { Taoism/ } \\
\text { Buddhism }\end{array}$ & 206 & 63.4 & No & 129 & 39.7 \\
\hline & Missing & 18 & 5.5 & & & \\
\hline \multicolumn{2}{|c|}{ Parameter } & Mean & SD & Parameter & Mean & SD \\
\hline \multicolumn{2}{|c|}{$\begin{array}{l}\text { Physical symptom } \\
\text { distress }\end{array}$} & 26.92 & 7.60 & $\begin{array}{l}\text { Perceived burden } \\
\text { to others }\end{array}$ & 24.45 & 10.08 \\
\hline \multicolumn{2}{|c|}{$\begin{array}{l}\text { Functional } \\
\text { dependence }\end{array}$} & 25.40 & 9.99 & Social support & 74.05 & 16.71 \\
\hline \multicolumn{2}{|c|}{$\begin{array}{l}\text { Psychological } \\
\text { symptom distress }\end{array}$} & 11.02 & 5.73 & & & \\
\hline
\end{tabular}

Our severe anxiety-symptom prevalence is within the range $(12.4 \%-34.0 \%)$ reported using the same HADS-A cutoff score for patients with advanced/ terminal cancer primarily under palliative care in the United States, ${ }^{1}$ Australia, ${ }^{9}$ The Netherlands, ${ }^{13}$ and Greece. ${ }^{8}$ We extend these results by showing the prevalence of severe anxiety symptoms not only in an Asian context but also longitudinally. The clinically important increase in severe anxiety-symptom prevalence as death approached observed in our unadjusted GEE analysis possibly reflects concerns about the unknown nature of impending death and its impact on loved ones. ${ }^{2,40}$ However, after controlling for covariates, time proximity to death per se was not significantly associated with severe anxiety symptoms, as concluded in studies indirectly measuring changes in anxiety symptoms at EOL, ${ }^{1,3,19}$ including a 94-study meta-analysis. ${ }^{3}$
The only demographic or disease-related characteristics associated with the likelihood of severe anxiety symptoms were diagnosis and metastatic status, consistent with conclusions from a 94-study metaanalysis ${ }^{3}$ and empirical studies. ${ }^{1,6,8}$ A lower likelihood of severe anxiety symptoms was seen among Taiwanese patients with lung and metastatic cancer at EOL, unlike previous reports. ${ }^{10,14}$ These conflicting results may be due to previous studies not controlling for the common plights of patients with lung or metastatic cancer, such as symptom distress, ${ }^{14}$ depressive symptoms, ${ }^{10}$ and functional dependence. ${ }^{10,14}$ Furthermore, Taiwanese patients with lung or metastatic cancer were suggested to be more likely to accurately understand their prognosis. ${ }^{41}$ Knowing one's poor prognosis facilitates coming to terms with one's mortality and decreases the likelihood of severe anxiety 
Tang et al

\begin{tabular}{|c|c|c|c|c|}
\hline Potential Predictor & Parameter & $95 \% \mathrm{Cl}$ & Z Score & $P$ Value \\
\hline \multicolumn{5}{|c|}{ Temporal proximity to patient death (d) } \\
\hline $1-30$ & 0.67 & $0.43,1.04$ & -1.77 & .08 \\
\hline $31-90$ & 0.85 & $0.57,1.26$ & -0.81 & .42 \\
\hline $91-180$ & 1.01 & $0.70,1.45$ & 0.05 & .96 \\
\hline $181-365$ & Reference & & & \\
\hline \multicolumn{5}{|l|}{ Disease-related characteristics } \\
\hline \multicolumn{5}{|l|}{ Cancer site } \\
\hline Lung & 0.43 & $0.21,0.89$ & -2.26 & .02 \\
\hline Breast & 0.45 & $0.18,1.15$ & -1.67 & .10 \\
\hline Colon/Rectum & 0.89 & $0.33,2.41$ & -0.23 & .82 \\
\hline Liver/Pancreas & 0.63 & $0.35,1.13$ & -1.54 & .12 \\
\hline Head and neck & 0.86 & $0.38,1.95$ & -0.36 & .72 \\
\hline Stomach & 0.61 & $0.35,1.05$ & -1.79 & .07 \\
\hline Other & Reference & & & \\
\hline \multicolumn{5}{|l|}{ Metastasis } \\
\hline Yes & 0.56 & $0.36,087$ & -2.59 & .01 \\
\hline No & Reference & & & \\
\hline \multicolumn{5}{|l|}{ Disease burden } \\
\hline Physical symptom distress & 1.12 & $1.09,1.14$ & 10.16 & $<.001$ \\
\hline \multicolumn{5}{|l|}{ Severe depressive symptoms } \\
\hline Yes & 4.55 & $2.94,7.14$ & 6.73 & $<.001$ \\
\hline No & Reference & & & \\
\hline \multicolumn{5}{|l|}{ Perceived burden to others } \\
\hline High & 1.75 & $1.32,2.31$ & 3.88 & $<.001$ \\
\hline Low & Reference & & & \\
\hline Social support & 1.02 & $1.01,1.03$ & 3.92 & $<.001$ \\
\hline
\end{tabular}

symptoms triggered by an unknown future. However, this hypothesis warrants further validation, because we did not explore the impacts of prognostic awareness and their interactions with a lung or metastatic cancer diagnosis on the likelihood of severe anxiety symptoms.

The likelihood of severe anxiety symptoms was significantly higher for participants with heavy disease burden, consistent with evidence linking anxiety to physical symptom distress ${ }^{5,7,8,10}$ and severe depressive symptoms. ${ }^{1,5,7,9}$ Our findings highlight the importance of managing both physical and emotional symptoms not only to relieve dying cancer patients' physical suffering but also to facilitate their psychological well-being. Our longitudinal data also confirm the common finding that anxiety and de- pression are highly correlated $d^{1,5,7,9}$ and comorbid ${ }^{2,3,21}$ but may evolve differently over the dying process. As for severe anxiety symptoms, we previously found that the raw severe depressive-symptom prevalence increased as death approached (44.58\%-82.64\% from 181-365 to 1-30 days before death). ${ }^{31}$ However, after confounding variables were controlled, severe depressive-symptom prevalence was significantly higher at 31 to 90 and 1 to 30 days relative to 91 to 180 days before death, contrasting with our current finding that these patients' severe anxietysymptom prevalence did not change significantly as death approached. Our findings suggest the need for longitudinal investigations using statistics such as latent growth mixture modeling to better understand the comorbid psychological distress of patients with 
Anxiety Symptoms at End of Life

cancer at EOL by identifying distinct classes for severe anxiety and depressive symptoms.

Our participants with high perceived burden to others were at elevated risk for severe anxiety symptoms. This result, based on longitudinal assessments of perceived burden to others until patient death, extends the cross-sectional association between anxiety and perceived burden to others. ${ }^{5}$ Taiwanese patients are likely to feel anxious when perceiving their family members' exhaustion from the substantial demands of their dying process and when receiving care without any opportunity to pay back, because this situation contradicts Asian views of interpersonal relationships, which function under the assumption of mutual obligation and responsibility. ${ }^{42}$ Feeling that one's relationships are reciprocal, harmonious, and positive is the basis of self-worth and self-esteem in Asian culture. Failure to fulfill such mutual obligations and responsibilities may provoke anxiety in Taiwanese/Asian patients with cancer at EOL.

Participants were more likely to experience severe anxiety symptoms if they perceived stronger social support, contrary to commonly reported findings. ${ }^{1,5,12}$ Social support functions as a buffer to regulate distress when people encounter imminent threats. ${ }^{43}$ The interpersonal fear of losing one's social identity over the dying process can be mitigated by forming and maintaining close relationships. ${ }^{44}$ However, facing death and anticipating the ultimate separation from loved ones to whom one is even more strongly connected in collectivistic Asian cultures than in individualized Western cultures may awaken feelings of separateness and evoke annihilation anxiety ${ }^{45}$ and aftermath concerns. Indeed, severing ties with or separating from loved ones through death is one of the most worrisome aspects of dying, ${ }^{46}$ and can increase a dying patient's likelihood of anxiety symptoms.

The strengths of this study lie in its longitudinal assessments of severe anxiety symptoms before and up to death in a large sample of patients with terminal cancer, using a validated multi-item screening instrument for anxiety, and exploring a range of potential correlates of severe anxiety symptoms as suggested. ${ }^{21}$ However, our findings should be interpreted with some caveats. Participants were recruited by convenience from a single medical center in Taiwan, limiting representativeness of the target population and generalizability of our results. A substantial proportion of patients $(10.5 \%)$ withdrew from the study, preventing generalization of our findings to those patients. Anxiety was not diagnosed by structured clinical interviews according to the Diagnostic and Statistical Manual of Mental Disorders, 4th Edition, possibly overestimating the prevalence of severe anxiety symptoms but avoiding the failure to recognize patients' need for psychological support. Associations of severe anxiety symptoms with disease burden, perceived burden to others, and social support do not indicate a cause-and-effect relationship. Furthermore, participants' severe anxiety symptoms may be due to unmeasured factors, such as discussion $^{6}$ and awareness with acceptance ${ }^{47}$ of prognosis. Participants' pre-enrollment anxiety symptoms or other mental health disorders (eg, alcohol problems) were not assessed, likely influencing their severe anxiety symptoms at EOL. Therefore, our observations might be anchored in situations preceding the dying process.

\section{Conclusions}

The severe anxiety-symptom prevalence among terminally ill Taiwanese patients with cancer increased as death approached. However, severe anxiety symptoms were not associated with temporal proximity to death nor with unchangeable demographic or diseaserelated characteristics, but were associated with factors that are modifiable by high-quality EOL care. Once advanced cancer status is recognized, the likelihood of severe anxiety symptoms at EOL may be decreased by integrating palliative care early in cancer care. ${ }^{48}$ Palliative care would better manage physical symptoms and provide psychological interventions to help patients. Evidence-based interventions ${ }^{21}$ with cognitive-behavioral or stress-management components may increase adaptive skills to cope with current and expected future stressors among patients with advanced cancer. Interventions with supportiveexpressive approaches may help terminally ill patients with cancer explore existential distress ${ }^{21}$ (eg, fear of their forthcoming death and anxiety about separation from loved ones) and accept unavoidable dependence on others' help at EOL, thus lightening perceived burden to others for those strongly connected with their social network. This last aspect is especially important for patients whose culture highly values reciprocal 
Tang et al

interpersonal relationships. Lightening this burden and extending psychological care to include anxiety may optimize the psychological well-being of dying patients with cancer.

\section{References}

1. Kolva E, Rosenfeld B, Pessin $\mathrm{H}$, et al. Anxiety in terminally ill cancer patients. J Pain Symptom Manage 2011;42:691-701.

2. Miovic M, Block S. Psychiatric disorders in advanced cancer. Cancer 2007;15:1665-1676.

3. Mitchell AJ, Chan M, Bhatti H, et al. Prevalence of depression, anxiety, and adjustment disorder in oncological, haematological, and palliativecare settings: a meta-analysis of 94 interview-based studies. Lancet Oncol 2011;12:160-174.

4. Spencer R, Nilsson M, Wright A, et al. Anxiety disorders in advanced cancer patients: correlates and predictors of end-of-life outcomes. Cancer 2010;116:1810-1819.

5. Wilson KG, Chochinov HM, Skirko MG, et al. Depression and anxiety disorders in palliative cancer care. J Pain Symptom Manage 2007;33:118-129.

6. Cripe LD, Rawl SM, Schmidt KK, et al. Discussions of life expectancy moderate relationships between prognosis and anxiety or depression in men with advanced cancer. J Palliat Med 2012;15:99-105.

7. Delgado-Guay M, Parsons HA, Li Z, et al. Symptom distress in advanced cancer patients with anxiety and depression in the palliative care setting. Support Care Cancer 2009;17:573-579.

8. Mystakidou K, Rosenfeld B, Parpa E, et al. Desire for death near the end of life: the role of depression, anxiety and pain. Gen Hosp Psychiatry 2005;27:258-262.

9. O'Connor M, White K, Kristjanson LJ, et al. The prevalence of anxiety and depression in palliative care patients with cancer in Western Australia and New South Wales. Med J Aust 2010;193(5 Suppl):S44-47.

10. Salvo N, Zeng L, Zhang L, et al. Frequency of reporting and predictive factors for anxiety and depression in patients with advanced cancer. Clin Oncol 2012;24:139-148.

11. Seow H, Barbera L, Sutradhar R, et al. Trajectory of performance status and symptom scores for patients with cancer during the last six months of life. J Clin Oncol 2011;29:1151-1158.

12. Stark D, Kiely M, Smith A, et al. Anxiety disorders in cancer patients: their nature, associations, and relation to quality of life. J Clin Oncol 2002;20:3137-3148.

13. Teunissen SC, de Graeff A, Voest EE, de Haes JC. Are anxiety and depressed mood related to physical symptom burden? A study in hospitalized advanced cancer patients. Palliat Med 2007;21:341-346.

14. Vodermaier A, Linden W, MacKenzie R, et al. Disease stage predicts postdiagnosis anxiety and depression only in some types of cancer. Br J Cancer 2011;105:1814-1817.

15. Warmenhoven F, van Weel C, Vissers K, Prins J. Screening instruments for depression in advanced cancer patients: what do we actually measure? Pain Pract 2013;13:467-475

16. McCoubrie RC, Davies AN. Is there a correlation between spirituality and anxiety and depression in patients with advanced cancer? Support Care Cancer 2005;14:379-385

17. Johnson KS, Tulsky JA, Hays JC, et al. Which domains of spirituality are associated with anxiety and depression in patients with advanced illness? J Gen Intern Med 2011;26:751-758.

18. Suarez-Almazor ME, Newman C, Hanson J, Bruera E. Attitudes of terminally ill cancer patients about euthanasia and assisted suicide: predominance of psychosocial determinants and beliefs over symptom distress and subsequent survival. J Clin Oncol 2002;20:2134-2141.

19. Lichtenthal WG, Nilsson M, Zhang B, et al. Do rates of mental disorders and existential distress among advanced stage cancer patients increase as death approaches? Psychooncology 2009;18:50-61.

20. Norton S, Cosco T, Doyle F, et al. The Hospital Anxiety and Depression Scale: a meta confirmatory factor analysis. J Psychosom Res 2013;74:74-81.

21. Traeger L, Greer JA, Fernandez-Robles C, et al. Evidence-based treatment of anxiety in patients with cancer. J Clin Oncol 2012;30:1197-1205.

22. Tang ST, Chang WC, Chen JS, et al. Trajectory and predictors of quality of life during the dying process: roles of perceived sense of burden to others and posttraumatic growth. Support Care Cancer 2014;22:2957-2964.

23. Zigmond AS, Snaith RP. The Hospital Anxiety and Depression Scale. Acta Psychiatr Scan 1983;67:361-370.
24. Wasteson E, Brenne E, Higginson IJ, et al. Depression assessment and classification in palliative cancer patients: a systematic literature review. Palliat Med 2009;23:739-753.

25. Mitchell AJ, Meader N, Symonds P. Diagnostic validity of the Hospital Anxiety and Depression Scale (HADS) in cancer and palliative settings: a meta-analysis. J Affect Disord 2010;126:335-348.

26. Carey M, Noble N, Sanson-Fisher R, MacKenzie L. Identifying psychological morbidity among people with cancer using the Hospital Anxiety and Depression Scale: time to revisit first principles? Psycho-Oncol 2012;21:229_ 238.

27. McCorkle R, Young K. Development of a symptom distress scale. Cancer Nurs 1978;1:373-378.

28. Benoliel JQ, McCorkle R, Young K. Development of a social dependency scale. Res Nurs Health 1980;3:3-10.

29. Stapleton SJ, Holden J, Epstein J, Wilkie DJ. A systematic review of the symptom distress scale in advanced cancer studies [published online ahead of print August 6, 2015]. Cancer Nurs, in press.

30. Tang ST, McCorkle R. A user's manual for the Enforced Social Dependency Scale. Available at: http://fhsson.mcmaster.ca/apn/images/stories/pdfs/Social_ Dependency_Scale_User's_Manual.pdf. Accessed February 14, 2016.

31. Tang ST, Chen JS, Chou WC, et al. Prevalence of severe depressive symptoms increases as death approaches and is associated with disease burden, tangible social support, and high self-perceived burden to others. Support Care Cancer 2016;24:83-91.

32. Cousineau N, McDowell I, Hotz S, Hebert P. Measuring chronic patient's feelings of being a burden to their caregivers: development and preliminary validation of a scale. Med Care 2003;41:110-118.

33. McPherson CJ, Wilson KG, Chyurlia L, Leclerc C. The balance of give and take in caregiver-partner relationships: an examination of self-perceived burden, relationship equity, and QOL from the perspective of care recipients following stroke. Rehabil Psychol 2010;55:194-203.

34. McPherson CJ, Wilson KG, Lobchuk MM, Brajtman S. Self-perceived burden to others: patient and family caregiver correlates. J Palliat Care 2007;23:135-142.

35. McPherson CJ, Wilson KG, Lobchuk MM, Brajtman S. Family caregivers' assessment of symptoms in patients with advanced cancer: concordance with patients and factors affecting accuracy. J Pain Symptom Manage 2008;35:7082.

36. Sherbourne CD, Stewart AL. The MOS Social Support Survey. Soc Sci Med 1991;32:705-714.

37. Shyu YIL, Tang WR, Liang J, et al. Psychometric testing of the Social Support Survey on a Taiwanese sample. Nurs Res 2006,55:411-417.

38. Christakis NA, Escarce JJ. Survival of Medicare patients after enrollment in hospice programs. N Engl J Med 1996;335:172-178.

39. Liang KY, Zeger S. Longitudinal data analysis using generalized linear models. Biometrika 1986;73:13-22

40. Kadan-Lottick NS, Vanderwerker LC, Block SD, et al. Psychiatric disorders and mental health service use in patients with advanced cancer. Cancer 2005;104:2872-2881.

41. Tang ST, Liu TW, Chow JM, et al. Associations between accurate prognostic understanding and end-of-life care preferences and its correlates among Taiwanese terminally ill cancer patients surveyed in 2011-2012. PsychoOncol 2014;23:780-787.

42. Kim HS, Sherman DK, Taylor SE. Culture and social support. Am Psychol 2008;63:518-526.

43. Cohen SE, Wills TA. Stress, social support, and the buffering hypothesis. Psychol Bull 1985;98:310-357.

44. Chochinov HM, Hack T, McClement $S$, et al. Dignity in the terminally ill: a developing empirical model. Soc Sci Med 2002;54:433-443.

45. Firestone RW. Individual defenses against death anxiety. Death Studies 1993;17:497-515.

46. Bath DM. Separation from loved ones in the fear of death. Death Studies 2010;34:404-425.

47. Thompson GN, Chochinov HM, Wilson KG, et al. Prognostic acceptance and the well-being of patients receiving palliative care for cancer. J Clin Oncol 2009;27:5757-5762.

48. Temel JS, Greer JA, Muzikansky A, et al. Early palliative care for patients with metastatic non-small-cell lung cancer. N Engl J Med 2010;363:733-742. 